\title{
Buschmann, Nikolaus, Planert, Ute, Vom Wandel eines Ideals. Bildung, Universität und Gesellschaft in Deutschland
}

\section{Marie-Bénédicte Vincent}

\section{CpenEdition}

Journals

Édition électronique

URL : http://journals.openedition.org/ifha/6680

DOI : $10.4000 /$ ifha. 6680

ISSN : 2198-8943

Éditeur

IFRA - Institut franco-allemand (sciences historiques et sociales)

Référence électronique

Marie-Bénédicte Vincent, «Buschmann, Nikolaus, Planert, Ute, Vom Wandel eines Ideals. Bildung, Universität und Gesellschaft in Deutschland», Revue de l'IFHA [En ligne], Date de recension, mis en ligne le 01 janvier 2011, consulté le 22 septembre 2020. URL : http://journals.openedition.org/ifha/6680 ; DOI : https://doi.org/10.4000/ifha.6680

Ce document a été généré automatiquement le 22 septembre 2020.

(CIFHA 


\title{
Buschmann, Nikolaus, Planert, Ute, Vom Wandel eines Ideals. Bildung, Universität und Gesellschaft in Deutschland
}

\author{
Marie-Bénédicte Vincent
}

1 « Humboldt est définitivement mort et enterré au début du XXIe siècle » : c'est le constat acerbe d'U. Frevert qu'on retiendra de ce petit volume collectif, publié à l'occasion du 65e anniversaire de l'historien Dieter Langewiesche, coéditeur entre autres du tome $5 \mathrm{du}$ Handbuch für Deutsche Bildungsgeschichte (1989). Sa propre biographie réunit les évolutions générales de la RFA en matière d'éducation : né en 1943 dans le monde ouvrier, il n'entre pas au Gymnasium, mais dans une Realschule de la Ruhr où il obtient à 16 ans le diplôme intermédiaire de la mittlere Reife, qui lui ouvre une formation commerciale. C'est grâce à des cours du soir à Gelsenkirchen qu'il réussit à passer l'Abitur en 1966 et à entrer à l'université de Heidelberg, dont il sort diplômé en histoire, germanistique et sciences politiques en 1971. Bénéficiant ensuite d'un poste d'assistant à l'université de Wurtzbourg, il peut entamer une thèse d'histoire. Langewiesche a ainsi profité de cette « seconde voie d'accès à la culture » dont parlait le sociologue Ralf Dahrendorf à la fin des années 1950, comme correctif et alternative permettant de réaliser l'ascension sociale en panne de la RFA (K. Schönhoven).

2 Le volume rassemble des auteurs célèbres autour de l'articulation difficile aujourd'hui entre la Bildung et l'université de masse en Allemagne. Mais l'hétérogénéité des contributions se fait sentir dès l'introduction qui se borne à juxtaposer leurs résumés. À la question de savoir comment concilier l'ouverture de l'Université, fondamentale pour l'efficience de la démocratie, et l'excellence scientifique, les auteurs se divisent en deux groupes. Les premiers s'interrogent sur les réformes universitaires en cours et reviennent sur les transferts entre l'Europe et les États-Unis : alors que l'Allemagne avait exporté outre-Atlantique son modèle universitaire vers 1900, elle a désormais les yeux rivés sur les établissements élitistes américains, au miroir desquels elle mesure 
ses propres déficiences (K.H. Jarausch, U. Frevert). Les seconds offrent un retour dans le passé pour montrer que certaines questions telles que " comment intégrer scolairement une minorité socialement et culturellement défavorisée ?" se posaient déjà sous le Kaiserreich : ainsi $\mathrm{S}$. Schraut relate l'histoire d'un succès, celui du rattrapage lors de la République de Weimar de "l'infériorité " des jeunes filles catholiques dans le système universitaire (le rapport entre étudiantes catholiques et protestantes est de 1 pour 10 dans les universités prussiennes en 1908, contre une pour 1,7 en 1925). Aujourd'hui se pose de manière aiguë la question de l'intégration scolaire des minorités issues de l'immigration. Un cinquième des jeunes Allemands de moins de 15 ans appartient à des Risikokategorien qui n'ont aucune chance de s'intégrer au marché du travail, rappelle H.-E. Tenorth. La société de masse connaîtrait une élévation paradoxale du niveau culturel de la population, réduite pourtant à vivre une situation de « demi-Bildung " selon $\mathrm{H}$. Bausinger, qui réfléchit à partir des quiz télévisés ou radiophoniques à mi-chemin entre divertissements et tests de connaissances. Ceci dit, le sens normatif du terme de Bildung est critiqué par K. Maase, qui voit en lui l'héritage d'un passé où le Bildungsbürgertum méprisait les médias de masse. Les contributions les plus réussies sont celles qui parviennent à mettre en perspective historique les réflexions actuelles. Mais l'ensemble reste inégal.

Marie-Bénédicte Vincent (université d'Angers, UMR 6258 CERHIO) 